\title{
Transport and Capacitance Spectroscopy of Quantum Dots
}

\author{
J. Adamowski, S. Bednarek and B. Szafran \\ Faculty of Physics and Nuclear Techniques, University of Mining and Metallurgy \\ Al. Mickiewicza 30, 30-059 Cracow, Poland
}

\begin{abstract}
A review of recent theoretical studies on a single-electron tunneling in quantum dots is presented. This effect underlies the transport spectroscopy performed on the vertical gated quantum dots and the capacitance spectroscopy on the self-assembled quantum dots. The conditions of the single-electron tunneling are formulated in terms of electrochemical potentials of the electrons in the leads and in the quantum dot. The electrochemical potentials for the electrons confined in the quantum dots can be calculated by solving the many-electron Schrödinger equation. The results obtained by the Hartree-Fock method are presented. For the vertical gated quantum dot, the realistic confinement potential is obtained from the Poisson equation. The application of the self-consistent procedure to the solution of the Poisson-Schrödinger problem is discussed. The calculated positions of the current peaks at zero bias and boundaries of the Coulomb diamonds for non-zero bias are in good agreement with experiment. The influence of an external magnetic field on the single-electron tunneling is also discussed. The spin-orbital configurations of the electrons confined in the quantum dots change with the magnetic field, which leads to features observed in the current-voltage and capacitance-voltage characteristics.
\end{abstract}

PACS numbers: 73.22.-f, 73.63.-b

\section{Introduction}

Excess electrons confined in quantum dots (QDs) form atomic-like states with discrete energy levels. The QD with the confined electrons can be treated as an artificial atom [1]. The potential that bounds electrons in the QD (confinement potential) is created by the band offsets at the interfaces of the QD and the external electric fields applied. This allows for a modelling of the electron energy spectrum of the artificial atoms. The energy levels of the artificial atoms can be 
detected in single-electron tunneling processes that form a basis of new spectroscopic tools: transport spectroscopy [2] and capacitance spectroscopy [3]. In the transport spectroscopy, the single-electron tunneling currents via the nanodevice are observed [4], while in the capacitance spectroscopy, the single-electron charging of QDs is detected as the changes of the differential capacitance [3]. Recently, the evidence has been found for the higher-order tunneling, the so-called cotunneling, in vertical gated QDs [5]. The transport experiments are usually performed on gated QDs [6, 7], i.e. three-terminal nanodevices with the gate electrode, which allows us to control the tunneling current. The experiments of Tarucha et al. [6] provide the beautiful evidence of the shell filling in the artificial atoms. The capacitance-spectroscopy measurements were done on self-assembled QDs [3, 8] in a magnetic field. These measurements [8] also yielded information about the electronic spectrum of the QDs.

In the present paper, we discuss theoretical methods applied to a description of the transport spectroscopy on the vertical gated QDs [6, 7] and capacitance spectroscopy on the self-assembled QDs [8]. In spite of the facts that the vertical gated and self-assembled QDs are fabricated by different nanotechnologies and the current and capacitance measurements are performed in different experimental setups [6, 8], they have the following common features: (i) the single-electron tunneling is the basic physical process underlying the transport and capacitance spectroscopy, which allows us to determine the spectra of the artificial atoms formed in both the types of QDs; (ii) the conditions for the observation of current and capacitance peaks as functions of the gate voltage are determined by the energetic balance between the electrochemical potentials of the electrons in the leads and the electrons confined in the QDs; (iii) both the types of the QDs possess the cylindrical rotational symmetry (this symmetry is almost perfect for the vertical gated QD and is a fairly good approximation of the shape of the self-assembled QD); (iv) the artificial-atom states formed in both the types of the QDs can be described by the Hartree-Fock method with the sufficient accuracy. In the present paper, we will exploit these common features to discuss the theory of the single-electron transport through the gated QDs [6] and the single-electron charging of the self-assembled QDs [8].

In the majority of theoretical papers [9-13], a two-dimensional model of the vertical gated $\mathrm{QD}$ was used with a parabolic confinement potential assumed. This model provides only a qualitative description of the experiments [6, 7]. The theoretical methods [14-16], presented in this article, are fully three-dimensional and apply more realistic confinement potentials. In particular, for the vertical gated QD we will discuss the solutions of the Poisson-Schrödinger problem for the entire nanodevice and show how they allow us to obtain the electron energy spectrum of the QDs in good agreement with experiment. We will also discuss the effect of an external magnetic field on the energy spectrum of QDs. 
The present paper is organized as follows: in Sec. 2 conditions of the single-electron tunneling are formulated; in Sec. 3 the problem of accuracy of the Hartree-Fock method is briefly discussed; in Sec. 4 the self-consistent procedure of the solution to the Poisson-Schrödinger problem is presented for the vertical gated QD; the computational results for the gated QDs are given in Sec. 5; in Sec. 6 the influence of the external magnetic field is discussed for both the gated and self-assembled QDs; in Sec. 7 we discuss the results of other theoretical methods; Sec. 8 contains conclusions, and Sec. 9 - a summary.

\section{Conditions of single-electron tunneling}

In order to observe either the single-electron currents via the $\mathrm{QD}$ or the change of the QD capacitance, we have to attach the leads to the nanodevice. The conditions of the single-electron tunneling are determined with the help of electrochemical potentials of the electrons in the leads and in the QD. The electrochemical potential of electrons in electrode $a$ of the nanodevice is defined as follows:

$$
\mu_{a}=\mu_{a}^{0}-e V_{a},
$$

where $\mu_{a}^{0}$ is the chemical potential of electrons in lead $a$ and $V_{a}$ is the voltage applied to $a$. For a metal electrode at zero temperature and zero voltage, the chemical potential defines the Fermi energy, i.e. $F_{a}=\mu_{a}^{0}$. The chemical potential of the QD that bounds $N$ excess electrons is defined as the energy needed to increase the number of electrons by one, i.e.

$$
\mu_{\mathrm{QD}}^{0}=\mu_{N+1}^{0}=E_{N+1}-E_{N},
$$

where $E_{N}$ is the ground-state energy of $N$ electrons confined in the QD.

In the gated QD, i.e. the nanodevice with the source (s), drain (d), and gate $(\mathrm{g})$ electrodes attached, the single-electron tunneling from the source to drain through the QD is energetically allowed if the following inequalities are fulfilled $[17,18]$ :

$$
\mu_{\mathrm{s}} \geq \mu_{N+1}^{0} \geq \mu_{\mathrm{d}},
$$

where $\mu_{\mathrm{s}}$ and $\mu_{\mathrm{d}}$ are the electrochemical potentials of the source and drain, respectively. In the gated QD, the source $\left(V_{\mathrm{s}}\right)$ and drain $\left(V_{\mathrm{d}}\right)$ voltages directly determine the corresponding electrochemical potentials (cf. Eq. (1)), while the gate voltage $\left(V_{\mathrm{g}}\right)$ affects the confinement potential, which changes the energy levels of the $N$-electron artificial atom, i.e. the chemical potential. This means that the gate voltage has an indirect, but essential influence on the single-electron tunneling. In this process, the number of electrons bound in the QD changes as follows: $N \longrightarrow N+1 \longrightarrow N \ldots$. Then, we speak about opening-up the transport window. If $F_{\mathrm{s}}=F_{\mathrm{d}}$, then - according to condition (3) - the single-electron transport is determined by the drain-source voltage $V_{\mathrm{ds}}=V_{\mathrm{d}}-V_{\mathrm{s}}$. Due to the weak inequalities in condition (3), the single-electron tunneling current can flow even at zero 
bias, i.e. at $V_{\mathrm{ds}}=0$. For the reverse bias the electrons flow from the drain to the source. In this case, the signs of inequalities in condition (3) have to be opposite. If condition (3) is not fulfilled, the number of electrons bound in the QD remains unchanged and we speak about the quantum Coulomb blockade [15]. The quantum Coulomb blockade results from the discreteness of the artificial-atom energy spectrum, which originates from the space quantization as well as the Coulomb repulsion between the electrons.

The electrons confined in self-assembled QDs are usually studied by the capacitance spectroscopy [8]. The capacitance spectroscopy is based on an application of a superposition of a dc voltage and small-amplitude ac voltage between the top and substrate contacts [8]. The condition for the single-electron tunneling can be formulated in terms of the electrochemical potentials as follows:

$$
\mu_{\mathrm{b}} \geq \mu_{N+1}
$$

where $\mu_{\mathrm{b}}$ and $\mu_{N+1}$ are the electrochemical potentials of the substrate (back) contact and $(N+1)$-electrons confined in the QD. Inequality (4) provides the condition for the single-electron tunneling from the substrate contact to the QD that contains $N$ excess electrons. If the sign of inequality (4) is opposite, the single electron can jump back from the QD to contact b. In the capacitance spectroscopy, the electrons do not reach the opposite (top) contact. This process, called the single-electron charging, leads to the oscillations of the charge confined in the QD, i.e. the change of the differential capacitance of the QD. These small changes of the capacitance can be detected by a high-resolution capacitance bridge [8].

\section{Hartree-Fock method}

The $N$-electron artificial atom is a quantum-mechanical system. Therefore, the ground-state energy of this system should be calculated by the quantum-mechanical methods. Usually, the authors apply either the density-functional method in the local density approximation (LDA) [10, 13, 19] or the Hartree-Fock (HF) method $[12,20]$. For few-electron systems, the configuration interaction (CI) method can be used $[9,11,21]$ (called "exact diagonalization"). In the LDA method, the electron-electron correlation is included in the local approximation, while in the HF method the correlation is neglected. The CI method allows for an incorporation of the correlation via the coupling between all allowed electron configurations. In Ref. [22], the problem of correlation was studied for two-electron systems in QDs with the harmonic-oscillator confinement potentials of both the spherical and cylindrical symmetry. It was shown [22] that - in small QDs - the HF method provides reliable results for the $N$-electron ground state. Only in large QDs, the HF results exhibit a few-percent relative error. For the QDs considered in this paper the estimated inaccuracy of the $\mathrm{HF}$ method is about $1 \mathrm{meV}$, which is less than the experimental uncertainty [6-8]. 
The HF equations were solved [14-16, 23] with the use of one-electron wave functions

$$
\psi_{\nu}(\boldsymbol{r})=\sum_{n} c_{n} g_{n}(\boldsymbol{r})
$$

expanded in the cylindrically-symmetric Gaussian basis

$$
g_{n}(\boldsymbol{r})=x^{p_{n}} y^{q_{n}} \exp \left[-\alpha_{n}\left(x^{2}+y^{2}\right)-\beta_{n} z^{2}\right]
$$

where $c_{n}, \alpha_{n}$, and $\beta_{n}$ are the variational parameters, $p_{n}$ and $q_{n}$ are integers $\left(p_{n}+q_{n} \leq 4\right)$. It was checked $[22,23]$ that basis $(6)$ yields accurate results for the spherical [23] as well as cylindrical [14-16] QDs.

\section{Poisson-Schrödinger problem for gated quantum dots}

The transport-spectroscopy experiments were performed on the vertical gated QDs [6]. The nanodevice [6] was made of the multi-layer $\mathrm{GaAs} / \mathrm{Al}_{0.22} \mathrm{Ga}_{0.78} \mathrm{As} /$ $\operatorname{In}_{0.05} \mathrm{Ga}_{0.95} \mathrm{As}$ heterostructure, which was etched to form a cylindrical pillar (Fig. 1a). The QD region within the InGaAs layer is defined by the two AlGaAs barriers that create the vertical confinement, and the ring-shaped Schottky gate that leads to the lateral confinement of the electrons. The nanodevice is terminated by the drain (top) and source (substrate) electrodes. The real nanostructure is asymmetric with respect to the inversion of the cylinder axis (cf. Fig. 1a). Nevertheless, the experimental data $[6,7]$ are nearly perfectly symmetric when reversing the polarity of the bias. Therefore, the (almost) symmetric model (Fig. 1b) of this nanostructure was applied in the papers $[14,15,24]$. The slightly different widths of the AlGaAs barriers are the only deviation from the inversion symme-
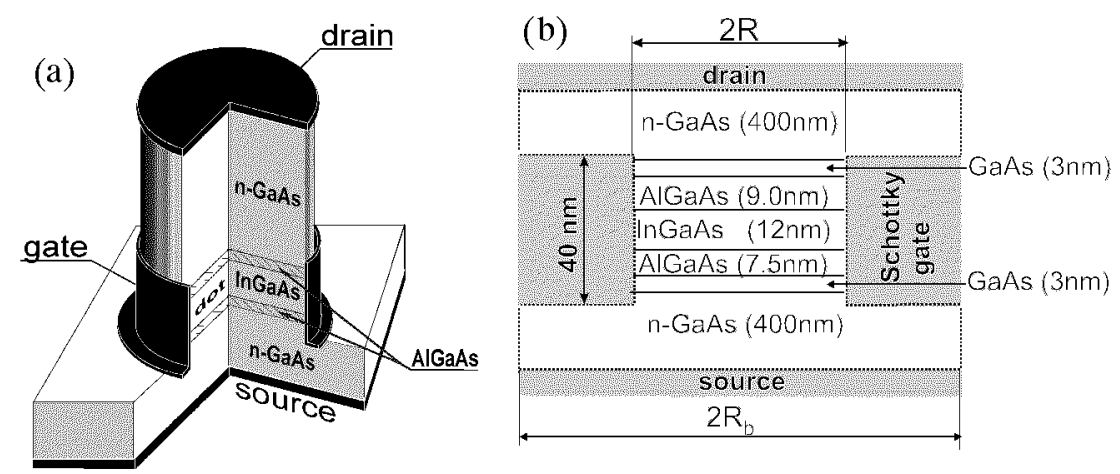

Fig. 1. (a) Schematic of the vertical gated QD fabricated by Tarucha et al. [6]. (b) Model nanostructure used in calculations [14, 15, 24]. Dotted line displays the cross section of the cylindrical surface, on which we put the boundary conditions for the Poisson equation. $R$ and $R_{\mathrm{b}}$ is the inner and outer radius of this surface, respectively. 
try with respect to the cylinder $(z)$ axis. On each side (source and drain) of the nanostructure (Fig. 1b), the $n$-GaAs layers are attached to the GaAs spacers. The application of the model nanostructure with the layers expanding up to infinity allowed us to put the boundary conditions for the Poisson equation [14, 15, 24].

In the gated QD, the excess electrons are confined by the confinement potential being the sum of the double-barrier vertical-confinement potential, which stems from the conduction-band offsets, and the electrostatic potential $\varphi_{1}(r)$, which is responsible for the lateral confinement of the electrons in the QD. Potential $\varphi_{1}(r)$ is a solution of the Poisson equation

$$
\nabla^{2} \varphi_{1}(r)=-\varrho_{\mathrm{D}}(r) / \varepsilon_{0} \varepsilon_{\mathrm{s}},
$$

where $\varrho_{\mathrm{D}}(r)$ is the space charge density originating from the ionized donors in the $n$-GaAs layers and $\varepsilon_{\mathrm{s}}$ is the static dielectric constant of GaAs [24]. In order to determine the total electrostatic field in the entire nanodevice [6] we have to take into account the additional electrostatic potential $\varphi_{2}(\boldsymbol{r})$, which results from the presence of $N$ excess electrons in the QD. Since these electrons form the artificial-atom states, they exert outside the QD the electrostatic field with the Hartree-type potential of the form

$$
\varphi_{2}(r)=-\frac{e}{4 \pi \varepsilon_{0} \varepsilon_{\mathrm{s}}} \sum_{\nu=1}^{N} \int \mathrm{d}^{3} r^{\prime} \frac{\left|\psi_{\nu}\left(\boldsymbol{r}^{\prime}\right)\right|^{2}}{\left|\boldsymbol{r}-\boldsymbol{r}^{\prime}\right|},
$$

where the sum runs over all the occupied one-electron states with wave functions $\psi_{\nu}(r)$. Therefore, the total electrostatic potential in the nanodevice is the sum

$$
\Phi_{\mathrm{tot}}(r)=\varphi_{1}(r)+\varphi_{2}(r) .
$$

In Eq. (7), the space charge density is associated with the ionized donors. At low temperature, we can neglect the thermal ionization of the donors; then the ionization of the donors is only caused by the electrostatic field with potential $\Phi_{\text {tot }}(r)$. In the nanodevice [6], the source and drain Ohmic contacts are made of the heavily doped $n$-GaAs layers. Therefore, in each part of the nanodevice, the donor energy level is aligned with the electrochemical potential of the source $\left(\mu_{\mathrm{s}}\right)$ and drain $\left(\mu_{\mathrm{d}}\right)$. In the $n$-GaAs layer, the ionization of the donor center at position $r$ occurs if the total potential energy of the electron

$$
U_{\text {tot }}(r)=-e \Phi_{\text {tot }}(r)
$$

exceeds the energy of the electron bound to the donor center, i.e. the corresponding electrochemical potential. This leads to the following donor ionization condition [24]:

$$
U_{\text {tot }}(\boldsymbol{r})>\mu_{\mathrm{s}(\mathrm{d})}
$$

for the source (s) and drain (d) side of the nanodevice. Condition (11) allows us to determine the space charge density as follows: if condition (11) is fulfilled, then $\varrho_{\mathrm{D}}(r)=e n_{\mathrm{D}}(\boldsymbol{r})$, and $\varrho_{\mathrm{D}}(\boldsymbol{r})=0$ otherwise. Here, we assume the donor concentration $n_{\mathrm{D}}(r)$ to be homogeneous in each $n$-GaAs layer. 
The boundary conditions for Poisson equation (7) are put on total potential (9) $[14,15,24]$ and the boundary values of potential $\varphi_{1}$ are calculated from Eq. (9). For the source and drain Ohmic contacts [6] the corresponding boundary conditions have the following form:

$$
\Phi_{\text {tot }}\left(\boldsymbol{r}_{\mathrm{s}(\mathrm{d})}\right)=V_{\mathrm{s}(\mathrm{d})},
$$

provided we take on the common Fermi energy of the leads $\left(F_{\mathrm{s}}=F_{\mathrm{d}}=F\right.$ ) as the reference energy, i.e. $F=0$. The metal gate adheres to the undoped semiconductor layers; therefore, we have to take into account the Schottky barrier of height $\phi_{\mathrm{B}}$, which leads to the following boundary condition at the gate surface:

$$
\Phi_{\mathrm{tot}}\left(r_{\mathrm{g}}\right)=V_{\mathrm{g}}-\phi_{\mathrm{B}} / \epsilon
$$

where $V_{\mathrm{g}}$ is the gate voltage.

The numerical solution to Poisson equation (7) in the cylindrical coordinates $(\rho, z)$ for the model nanostructure (Fig. 1b) yields the values of potential $\varphi_{1}$ on the mesh, i.e. $\varphi_{1}\left(\rho_{i}, z_{j}\right)$. The total confinement potential is the sum of $\varphi_{1}$ and the double-barrier potential with the thin potential well, which determines the extension of the QD in the vertical $(z)$ direction. Since potential $\varphi_{1}(\rho, z)$ very weakly changes with $z$ within the $\mathrm{QD}$, it can be approximated $[14,24]$ by its values at $z=0$. For the further application in the HF method, it is convenient to adjust an analytical formula to numerical solutions $\varphi_{1}\left(\rho_{i}, 0\right)$. It was found [14] that the six-order polynomial

$$
\widetilde{\varphi_{1}}(\rho)=\sum_{k=0}^{3} v_{k} \rho^{2 k}
$$

provides the accurate fit required.

The calculated confinement potential energy $U_{1}=-e \varphi_{1}$ of the electron is depicted in Figs. 2 and 3. Figure 2 shows the results for $N=12$ electrons confined in the QD, $V_{\mathrm{ds}}=0$, and $V_{\mathrm{g}}=-1 \mathrm{~V}$. Six-order polynomial (14) (solid curve) has been adjusted to the numerical solutions (full circles) in the interval $0 \leq \rho \leq R$, where $R$ is the inner radius of the gate. The dashed curve shows the parabolic fit to the numerical solutions. This fit is valid below the Fermi level (solid horizontal straight line in Fig. 2). The charge density of the electrons confined in the QD is shown by the thin solid curve. We note that the lateral confinement potential energy $U_{1}(\rho)$ can be fairly well approximated by the parabola below the Fermi level, i.e. in this region of the QD, in which the electrons are localized. The non-parabolic corrections start to play a role near and above the Fermi energy. However, the incorporation of the non-parabolicity of the lateral confinement potential is important for the accurate quantitative description [14, 24] of the transport-spectroscopy data $[6,7]$. The properties of the one- and two-electron energy spectra in the non-parabolic (Gaussian) confinement potential were studied in Ref. [25]. 


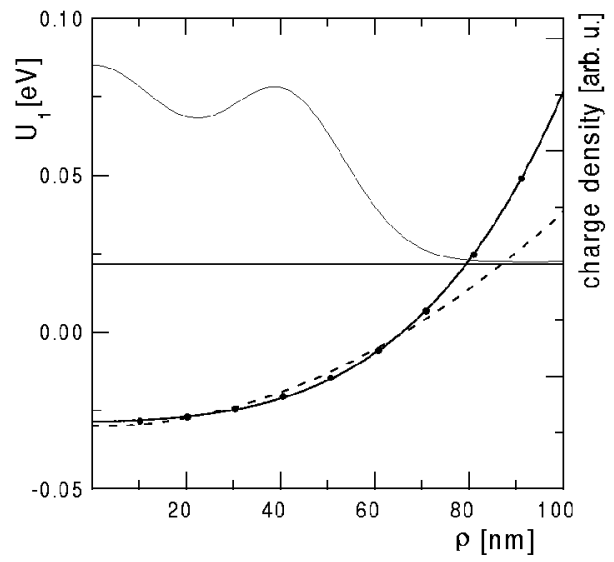

Fig. 2. Potential energy $U_{1}=-e \varphi_{1}$ (dots - numerical solutions of the Poisson equation, solid curve - fitted six-order polynomial, dashed curve - parabola fitted to the numerical solutions below the Fermi energy) and charge density (thin solid curve) for 12 electrons confined in the QD as functions of cylindrical coordinate $\rho$. Solid horizontal line corresponds to the Fermi energy. The curves are plotted for $V_{\mathrm{g}}=-1 \mathrm{~V}$ and $V_{\mathrm{ds}}=0$.

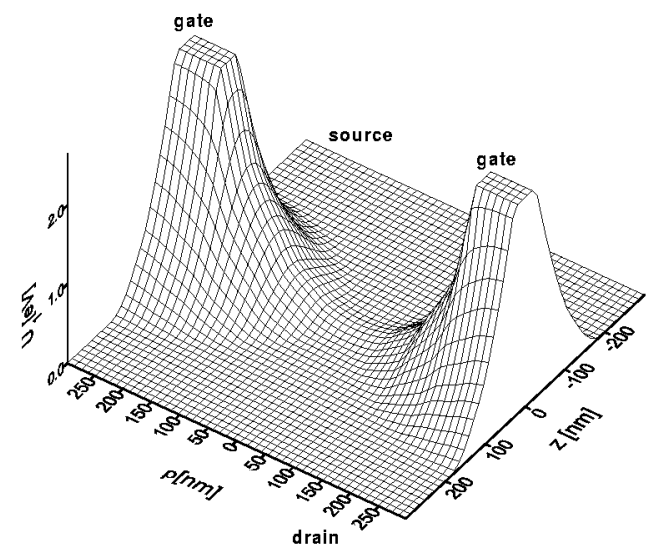

Fig. 3. Quasi-three-dimensional plot of the potential energy $U_{1}=-e \varphi_{1}$ in the nanodevice of Tarucha et al. [6]. $\rho$ and $z$ are the cylindrical coordinates.

In Fig. 3 the quasi-three-dimensional profile of the potential energy $U_{1}(\rho, z)$ is displayed for the entire nanodevice. The confined electrons are localized in the central region of the nanodevice, i.e. near $\rho \simeq 0$ and $z \simeq 0$. We note that potential energy $U_{1}$ is strongly dependent on the gate voltage $V_{\mathrm{g}}$ and number $N$ of electrons confined in the QD $[14,24]$. 


\section{Theoretical interpretation of transport spectroscopy results for cylindrical gated quantum dots}

The solutions of Poisson equation (7) provide the realistic confinement potential, which together with the double-barrier potential was used $[14,15,24]$ in the $\mathrm{HF}$ method as an external potential in order to calculate the ground-state energy $\left(E_{N}\right)$ for $N$ electrons bound in the QD. Since the relevant quantities, i.e. potential $\varphi_{1}$ and one-electron wave functions $\psi_{\nu}$, are coupled through Eqs. (8) and (9), the Poisson and Hartree-Fock equations have to be solved by a self-consistent procedure $[14,24]$. As a result, we obtain $[14,24]$ the chemical potential for the $N$-electron artificial atom as a function of the gate voltage (Fig. 4). In Fig. 4 the

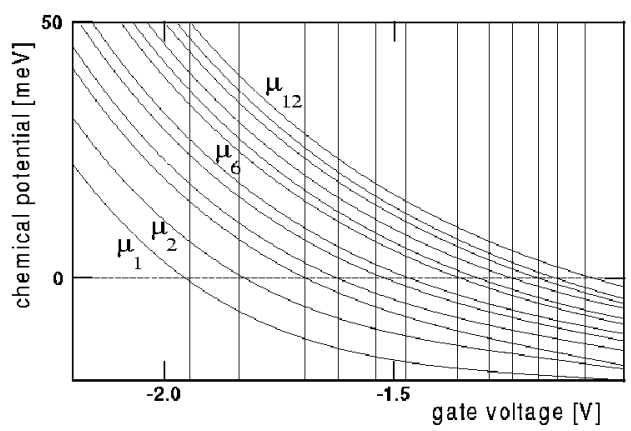

Fig. 4. Calculated chemical potential $\mu_{N}$ as a function of the gate voltage and number $N$ of QD-confined electrons for $V_{\mathrm{ds}}=0$. Thin vertical lines mark the positions of the single-electron tunneling current peaks measured [6] at $V_{\mathrm{ds}} \simeq 0$. The zero on the energy scale is set at the common source-drain Fermi level (horizontal line).

zero on the energy scale corresponds to the common Fermi energy of the source and drain $\left(F_{\mathrm{s}}=F_{\mathrm{d}}=F\right)$. For $V_{\mathrm{ds}}=0$ the single-electron tunneling occurs if $\mu_{N+1}^{0}=F$ (cf. condition (3)). Therefore, in Fig. 4 the crossing points of the chemical-potential plots with the abscissa determine the positions of the tunneling-current peaks [6] on the gate-voltage scale. In Fig. 4 the thin vertical lines mark the positions of the current peaks measured by Tarucha et al. [6] at $V_{\mathrm{ds}} \simeq 0$. The agreement between the theoretical $[14,24]$ and experimental results [6] is very good. Each peak of current results from the tunneling of the single electrons via the QD, while the lack of current (intervals between the peaks) corresponds to the fixed number of the electrons confined in the QD, i.e. the quantum Coulomb blockade [15]. The spacings between the current peaks are not equal to each other, which is a quantum-mechanical effect, caused by the filling of the subsequent electronic shells of the artificial atoms.

Kouwenhoven et al. [7] also reported the measurements of the differential conductance of the nanodevice as a function of the drain-source voltage and 


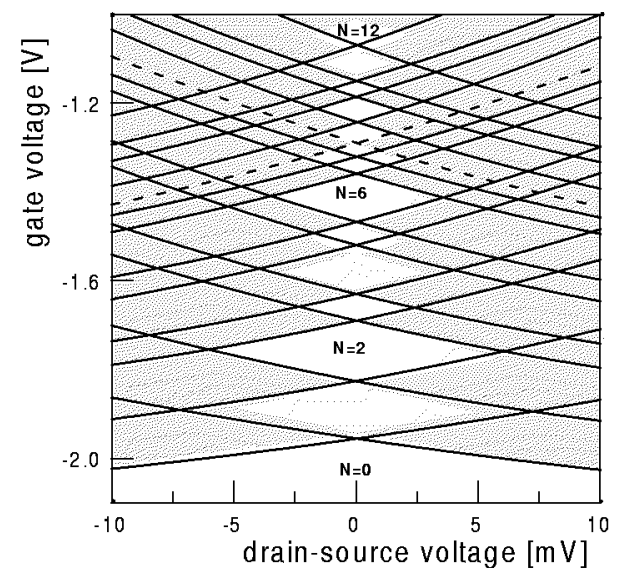

Fig. 5. Stability diagram with Coulomb diamonds on the gate voltage-drain-source voltage plane. Shaded (white) areas correspond to non-zero (zero) differential conductance [7]. Solid curves show the calculated [14, 24] boundaries of the single-electron tunneling via the $N$-electron ground state, dashed curves show these boundaries for the first excited state of the 8-electron artificial atom. In white, diamond-shaped regions, the number of electrons confined in the QD is fixed and equal to $N$.

gate voltage and obtained the stability diagram with the diamond-shaped regions, which are the signatures of the quantum Coulomb blockade. In Fig. 5 the white (shaded) areas correspond to the zero (non-zero) conductance, determined in the experiment [7]. The boundaries of the Coulomb diamonds, calculated according to condition (3), are shown by solid lines. The curves of the positive and negative slope result from the condition: $\mu_{N+1}^{0}=\mu_{\mathrm{s}}$ and $\mu_{N+1}^{0}=\mu_{\mathrm{d}}$, respectively. In Fig. 5 the solid curves have been obtained for the single-electron tunneling via the $N$-electron ground state and the dashed curves have been calculated under assumption of the tunneling via the first excited state of the eight-electron artificial atom [24]. Figure 5 shows that the calculated [24] positions, sizes, and shapes of the twelve Coulomb diamonds very well agree with the experimental results [7].

\section{Single-electron tunneling in a magnetic field}

\subsection{Vertical gated quantum dots}

In Refs. $[14,15,26]$ the approach presented in Sec. 4 was applied to a description of the magnetic-field behavior of the vertical gated QDs. The results $[14,15]$ are depicted in Fig. 6, on which solid (dashed) curves show the calculated (measured) gate voltage, for which the $N$-th electron tunnels through the QD at zero bias in the magnetic field applied in the vertical direction. The number of electrons confined in the QD is constant in the regions between the curves in Fig. 6. In these 


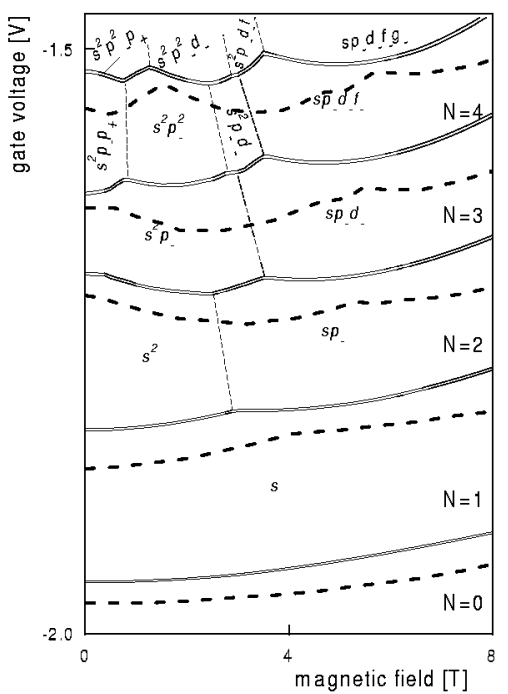

Fig. 6. Calculated [14] (solid curves) and measured [6] (dashed curves) gate voltage, at which the single electron tunnels via the gated $Q D$ at zero bias as a function of the magnetic field. The number $N$ of electrons confined in the QD is fixed in the areas between the curves. Occupied one-electron orbitals are denoted by the symbols described in the text. Thin dashed lines connect the cusps that correspond to the ground-state transformations in the $N$-electron artificial atom.

regions, the quantum Coulomb blockade occurs [15]. In Fig. 6 the one-electron orbitals occupied by the electrons are denoted by the symbols $s, p_{ \pm}, d_{ \pm}, f_{ \pm}$, and $g_{ \pm}$, which correspond to the magnetic quantum number $m=0, \pm 1, \pm 2, \pm 3$, and \pm 4 , respectively. The results of calculations $[14,15]$ fairly well agree with the experimental data $[6,7]$.

In Fig. 6 the cusps, connected by the thin dashed line, correspond to the magnetic-field induced ground-state transformation in the $N$-electron artificial atom. This double observation of the ground-state transformation results from condition (3) of the single-electron tunneling, which is determined by the chemical potential, being the difference of the ground-state energies of the $(N+1)$ - and $N$-electron systems. Therefore, each ground-state transformation is observed twice as the cusps of the curves, which correspond to the tunneling of the $(N+1)$-th and $N$-th electron through the QD. For each $N$ the same ground-state transformation appears at different magnetic fields, because the confinement potential changes with the gate voltage $[14,15]$ applied to the gated QD. For the larger gate voltage the $N$-electron system is more localized as the effect of the stronger confinement. Therefore, the change of the electronic configuration, which is associated with the occupation of the high-orbital momentum states, requires the weaker magnetic field. 


\subsection{Self-assembled quantum dots}

The capacitance-spectroscopy measurements were performed by Miller et al. [8] on the self-assembled QDs in an external magnetic field. The InAs QDs were grown on the GaAs substrate in a Stranski-Krastanow growth mode. The QDs obtained [8] were nearly uniform in size with an average radius $10 \mathrm{~nm}$ and height $7 \mathrm{~nm}$. The nanostructure [8] consists of the back (substrate) contact, made of the heavily doped GaAs, the undoped GaAs tunnel barrier, the InAs wetting layer with InAs self-assembled QDs, the AlAs/GaAs blocking barrier, and the
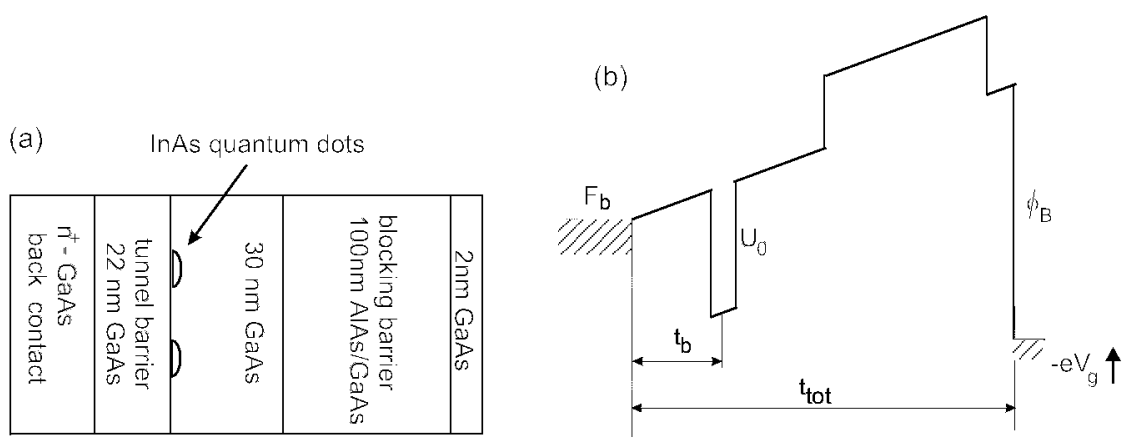

Fig. 7. (a) Layer sequence in the nanodevice of Miller et al. [8]. The self-assembled InAs QDs are distributed on the InAs wetting layer (not shown) between two GaAs layers. (b) Sketch of the vertical-confinement potential energy along the growth direction for gate voltage $V_{\mathrm{g}}$, for which the $\mathrm{QD}$ is empty. $t_{\mathrm{b}}$ and $t_{\mathrm{tot}}$ denote the distance between the QD and the back contact and the total thickness of the nanodevice, respectively.

GaAs gate (top) contact (Fig. 7a). The single-electron charging of the QD was tuned with the voltage $\left(V_{\mathrm{g}}\right)$ applied between the top (gate) and back contact (Fig. 7b). The voltage, measured along the growth direction, linearly changes with the distance from the substrate (Fig. 7b). If $t_{\mathrm{b}}$ is the distance from the back contact to the QD layer and $t_{\text {tot }}$ is the distance between the back and gate contact, fraction $\lambda=t_{\mathrm{b}} / t_{\text {tot }}$ of the gate voltage is directly applied to the $\mathrm{QD}$, changing the electrochemical potential of the QD. For the self-assembled QDs [8] the condition of the single-electron charging of the QD has the form (cf. Eq. (4))

$$
F_{\mathrm{b}}=\mu_{N+1}^{0}+\lambda\left(\phi_{\mathrm{B}}-e V_{\mathrm{g}}\right)
$$

where $F_{\mathrm{b}}$ is the Fermi energy of the back contact and $\phi_{\mathrm{B}}$ is the Schottky barrier at the gate/blocking barrier interface (cf. Fig. $7 b$ ). Equation (15) determines the positions of the capacitance peaks and additionally enables us to convert the energy into the gate-voltage scale.

In the self-assembled QDs, the confinement potential originates from the band-edge discontinuities. The shape of the QD can be approximated by the cylinder. Accordingly, in the calculations [16], the electron confinement potential energy was assumed to have the following cylindrically symmetric form: 


$$
U(\rho, z)= \begin{cases}U_{0} & \text { for } \rho<R \text { and }|z|<H / 2 \\ 0 & \text { otherwise }\end{cases}
$$

where $U_{0}$ is the conduction-band offset at the GaAs/InAs interface, $R$ is the radius of the QD, and $H$ is its height. For the self-assembled QDs [8] the assumption of the confining potential to be independent of the gate voltage as well as the number of electrons in the QD is well justified. Using (16) as the external potential energy, the $N$-electron Schrödinger equation was solved [16] by the unrestricted HF method with the Gaussian basis (6). This allowed us [16] to determine the chemical potential $\left(\mu_{N}^{0}\right)$ of the electrons confined in the QD. The chemical potential was converted into the gate voltage with the help of Eq. (15).

The measured [8] and calculated [16] gate voltage corresponding to the capacitance peaks is plotted in Fig. 8 as a function of the magnetic field. In Fig. 8 each pair of curves (solid and dashed) corresponds to $N$ electrons confined in the QD. The cusps on the curves are the signatures of the magnetic-field induced phase transitions [16]. For example, the cusp for $N=5$ results from the change of spin-orbital $s^{2} p_{-}^{2} p_{+}$with the total magnetic quantum number $M=-1$ into $s^{2} p_{-}^{2} d_{-}$with $M=-4$. The low-field phase transitions (at $\sim 1 \mathrm{~T}$ ) were not detected in experiments [8]. Nevertheless, the theoretical [16] and experimental plots [8] as well as the positions of the high-field phase transitions agree very well with each other.

In the capacitance spectroscopy on the self-assembled QDs [8] — similarly as in the gated QDs - the $N$-electron ground-state transformation can also be observed on the two plots corresponding to $N$ and $N+1$ electrons confined in the QD (Fig. 8). Since in the self-assembled QDs the confining potential is independent of the gate voltage, the corresponding cusps are located at the same magnetic field, denoted by arrows in Fig. 8. Because the experimental data (dashed curves in Fig. 8) have been extracted from rather broad capacitance peaks [8], this effect is weakly visible in experiment [8].

The quantitative discussion of the applicability of the quasi-two-dimensional model of the self-assembled QD was another interesting outcome of Ref. [16]. It was shown [16] that the self-assembled QDs of the comparable height and radius cannot be treated as quasi-two-dimensional with electrons frozen in the ground state, which is associated with the space quantization of the electron motion in the $z$ direction. If the height of the dot exceeds the dot radius, the excited one-electron states, associated with the vertical space quantization, start to play a role, which changes the symmetry of the $N$-electron wave function and strongly affects the magnetic-field behavior [16]. The theoretical predictions [16] have not yet been confirmed by experiments.

\section{Discussion}

Several theoretical papers $[9-15,20,27,28]$ were addressed to the electronic properties of vertical gated QDs [6]. The authors of Refs. [9-13, 28] assumed the 


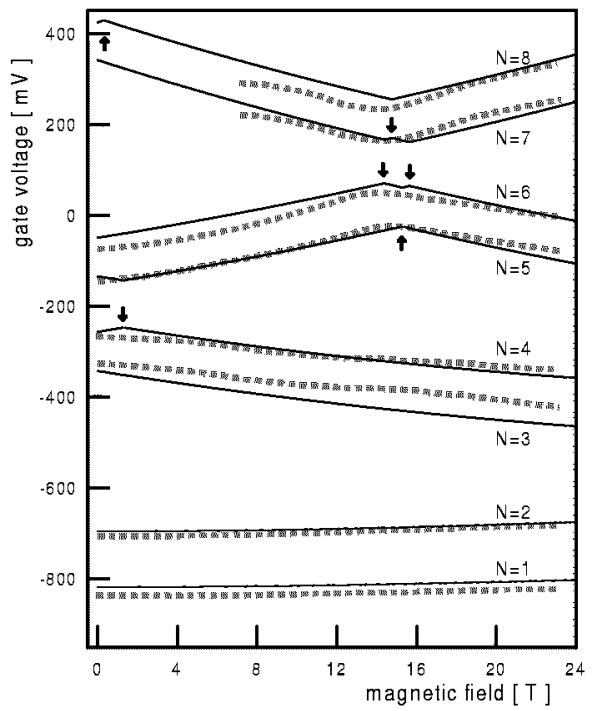

Fig. 8. Calculated [15] (solid curves) and measured [8] (dashed curves) gate voltage, at which the self-assembled QDs are charged by the $N$-th electron, as a function of the magnetic field. The arrows show the magnetic fields, at which the ground-state transformation occurs in the $N$-electron artificial atom.

model confinement potential, which was independent of the gate voltage and the number of electrons confined in the QD. Usually, it was the harmonic-oscillator potential in the two [9-10, 12, 13] and three [20] dimensions. The anisotropic harmonic-oscillator potential was used in Refs. [11, 19, 28]. The application of such fixed confining potentials led to a qualitative description of shell filling observed at zero bias [6]. The results of papers [14, 15, 24] show that - below the Fermi level - the realistic confinement potential can be fairly well approximated by the parabola (cf. Fig. 2), which explains why the simple parabolic approximation leads to the qualitatively correct results. However, the accurate quantitative description requires the electrostatics of the entire nanodevice to be taken into account. In the papers $[11-13,19,20,28]$ an addition energy was calculated. This quantity, defined as the difference between the chemical potentials, i.e. $\Delta \mu_{N}=\mu_{N+1}-\mu_{N}$, quite well shows the effects of shell filling, but cannot be directly compared with experiment [6]. In order to compare the addition energy with experiment, one has to know a factor that converts the energy scale into the gate-voltage scale [29]. As shown in Ref. [24] (cf. also Fig. 4), the conversion factor strongly depends on the gate voltage and number of electrons confined in the QD. These dependences are neglected when using the fixed confining potentials [9-13, 19, 20, 28].

The Poisson equation was solved for the confining potential in gated QDs in Refs. [21] and [27]. Nagaraja et al. [27] introduced a parallelepiped model for the 
cylindrical nanostructure of Tarucha et al. [6] and obtained the addition energy in qualitative agreement with that energy extracted from the experiment [6]. The lack of the proper symmetry and the undetermined conversion factor disabled the authors [27] to obtain the quantitative agreement. Bruce and Maksym [21] considered the another type of the nanodevice, made by Ashoori et al. [30]. These authors [21] solved the Schrödinger equation for the fixed number $(N=3)$ of electrons in the QD and the Poisson equation for the model nanostructure using the Green function method. The screening of the electron-electron interaction within the QD was taken into account with the help of image charges. This approach is equivalent to that presented in Sec. 4 and Refs. [14, 15, 24]. The method elaborated by the present authors $[14,15,24]$ seems to be simpler, which allows us to perform more extensive calculations with a larger number of electrons. In this method (Sec. 4), all the charges in the nanodevice are included via the ionized-donor charge density and boundary conditions put on the total electrostatic potential (Eq. (9))]. This leads to the screening, which is dependent on the gate voltage and number of electrons confined in the QD, which in turn gives rise to the realistic confinement potential.

The vertical gated QDs [6] are fabricated by the technological process that includes the combination of wet and dry etching of the layer AlGaAs/InGaAs/GaAs heterostructure. The side surface of the cylindrical pillar, obtained after this process, can be rather rough and can contain many defects. These imperfections give rise to a fluctuating potential near the surface. It arises a question: how does the fluctuating potential of the surface affect the electronic properties of the QD? A partial answer to this question can be given on a basis of the recent theoretical study [31] of clean and disordered QDs. It was shown [31] that the clear picture of the shell filling is obtained for the clean QDs, while the randomly distributed impurity potentials considerably disturb the spectrum of the QD. The transport-spectroscopy results for the gated QDs $[6,7]$ are quite regular with sharp current peaks (cf. Figs. 4 and 5). Based on the regular pattern of the current and conductance peaks $[6,7]$, we can state that the fluctuating potentials, created by the defects on the cylinder surface, have negligible influence on the electronic properties of QDs. The range of the fluctuating potential that stems from the remote surface of the pillar is too small to be effective in this region of the QD, in which the confined electrons are localized (cf. Fig. 2).

An attempt of a theoretical description of the capacitance-spectroscopy results [8] for the self-assembled QDs was undertaken by Warburton et al. [32] with the use of the two-dimensional parabolic confining potential. The calculations [32] were performed with the two fitting parameters, i.e. the oscillator energy and oscillator length, which were treated as independent of each other. As a result, the authors [32] separately treated the energy and length scales in the harmonic-oscillator problem, which is completely unjustified. As discussed in Ref. [16], the two-dimensional model is too poor for the self-assembled QDs; 
moreover, the breaking of the generalized Kohn theorem [33, 34], observed in the self-assembled QDs [35], suggests that the confinement potential significantly differs from the parabolic potential. For the self-assembled QDs, the model confining potentials in the form of the finite rectangular potential well [15] and the Gaussian potential [25] much better account for the conduction-band offset at the InAs/GaAs interface and the varying composition within the InGaAs dots [36].

\section{Conclusions}

The transport- and capacitance-spectroscopy methods allow us to determine the energy spectrum of QD-confined electrons with different accuracy. The accuracy of the transport spectroscopy performed on a single gated QD [6] is considerably larger than that of the capacitance spectroscopy made on randomly distributed self-assembled QDs [8]. In the latter case, the accuracy is limited by the random distribution of the QDs, size fluctuations, and interdot coupling. In the transport spectroscopy, the tunneling current peaks detected as functions of the gate voltage at zero bias are narrow [6], which allows us to extract from their positions the energy levels of artificial atoms with an uncertainty of a few meV. On the contrary, the peaks of the differential capacitance [8] are rather broad and the estimated uncertainty of these measurements is about $5 \mathrm{meV}$ [8].

The conditions of the single-electron tunneling (Eqs. (3) and (4)) involve the chemical potential, which was calculated in Refs. [14-16] by the HF method. The HF method is based on the quantum-mechanical variational principle; therefore, it provides upper bounds on the true energy of the considered system. The systematic overestimation of the energy in the HF calculations results from the neglected electron-electron correlation. For the QDs of the small size [6, 8] the error of the HF upper bounds on the ground-state energy was estimated [22] to be about $1 \mathrm{meV}$. Therefore, this error is smaller than the estimated uncertainty of both the transport [6] and capacitance [8] spectroscopy experimental results.

The HF estimates can be improved when using the CI method. Due to its computational complexity, the CI calculations were mainly performed in the framework of the two-dimensional model of the QDs [9, 11]. The LDA approach provides another possible method for taking into account the correlation in QDs $[10,13,19,28]$.

The nanodevice [6] with the gated QD is a prototype of a single-electron transistor (SET) [37]. Figures 4 and 5 illustrate the operation of the SET at zero and non-zero bias, respectively. When varying the gate voltage the subsequent transport windows are opened and closed, which corresponds to the SET being either in the on- or off-state. Contrary to the conventional transistor, the SET can be switched on and off at discrete gate-voltage values. At low temperature, the operation of SET can be tuned by the external voltages with a high precision. However, at the present stage of the nanotechnology, the direct application of the gated QD [6] to the room-temperature SET operation seems to be rather difficult. 
The results of Refs. $[14,15,24]$ are based on the stationary treatment of the single-electron tunneling via the QDs. We would like to comment on the applicability of this approach to this essentially dynamic process. The physical interpretation of single-electron tunneling conditions (3) and (4) is the following: these conditions determine the borders between the subsequent Coulomb blockade regions (Figs. 4 and 5). The quantum Coulomb blockade [15] is associated with the formation of the stationary quantum state of the $N$-electron artificial atom. If either the gate voltage or the drain-source voltage crosses the borders determined by conditions (3) and (4), then - as a result of the single-electron tunneling - the $N$-electron system makes an abrupt transition into the $(N+1)$-electron stationary state. This allows us to determine the positions of current and capacitance peaks from conditions (3) and (4). We note that this approach to the single-electron tunneling spectroscopy on the artificial atoms is similar to that used in the interpretation of the optical-spectroscopy data for the natural atoms. The single-electron tunneling via the QDs is an analog of the ionization of the natural atoms. In particular, the addition energy $\left(\Delta \mu_{N}\right)$ can be expressed as the difference between the ionization energy and electron affinity [29]. A possible time-dependent approach to the single-electron tunneling in QDs should result in a calculation of the heights and shapes of the current and capacitance peaks.

\section{Summary}

In Refs. [14, 15, 24], a theoretical method has been proposed for a description of basic stationary electronic properties of the vertical gated QD [6, 7]. In this approach $[14,15,24]$, the confinement potential was not assumed as in the other papers $[9-13,19,20,28]$, but calculated from the first principles of electrostatics, which led to a realistic profile of this potential. This calculated confinement potential was used in the $\mathrm{HF}$ method as the external potential for the electrons confined in the QD. Since the quantum states of the confined electrons affect the ionized donor distribution, i.e. the external confining potential, the resulting Poisson-Schrödinger problem had to solved in a self-consistent manner. The self-consistent procedure, elaborated in Refs. [14, 15, 24], enabled us to obtain the complete quantitative description of the spectacular transport-spectroscopy results $[6,7]$. To the best of our knowledge, only in Refs. [14, 15, 24], the shapes, sizes, and positions of the Coulomb diamonds on the stability diagram [7] were reproduced in the framework of the quantum-mechanical theory.

In the future the method $[14,15,24]$ can be applied to QDs of arbitrary geometry and may be extended to a description of the recently observed cotunneling [5] and Kondo effect [38]. 


\section{Acknowledgments}

We are grateful to Leo Kouwenhoven, Guy Austing, and Seigo Tarucha for stimulating scientific discussions and for providing us with material parameters of the vertical gated quantum dot. This work has been partly supported by the State Committee for Scientific Research under grant No. 5P03B 4920.

\section{References}

[1] P.A. Maksym, T. Chakraborty, Phys. Rev. Lett. 65, 108 (1990); M.A. Kastner, Phys. Today 16, 24 (1993).

[2] U. Meirav, P.L. McEuen, M.A. Kastner, E.B. Foxman, A. Kumar, S.J. Wind, Z. Phys. B, Condens. Matter 85, 357 (1991).

[3] R.C. Ashoori, H.L. Stormer, J.S. Weiner, L.N. Pfeiffer, S.J. Pearton, K.W. Baldwin, K.W. West, Phys. Rev. Lett. 68, 3088 (1992).

[4] T. Schmidt, M. Tewordt, R.H. Blick, R.J. Haug, D. Pfannkuche, K. v. Klitzing, A. Förster, H. Lüth, Phys. Rev. B 51, 5570 (1995).

[5] S. De Franceschi, S. Sasaki, J.M. Elzerman, W.G. van der Wiel, S. Tarucha, L.P. Kouwenhoven, Phys. Rev. Lett. 86, 878 (2001).

[6] S. Tarucha, D.G. Austing, T. Honda, R.J. van der Hage, L.P. Kouwenhoven, Phys. Rev. Lett. 77, 3613 (1996).

[7] L.P. Kouwenhoven, T.H. Oosterkamp, M.W.S. Danoesastro, M. Eto, D.G. Austing, T. Honda, S. Tarucha, Science 278, 1788 (1997).

[8] B.T. Miller, W. Hansen, S. Manus, R.J. Luyken, A. Lorke, J.P. Kotthaus, S. Huant, G. Medeiros-Ribeiro, P.M. Petroff, Phys. Rev. B 56, 6764 (1997).

[9] M. Eto, Jpn. J. Appl. Phys. 36, 3924 (1997).

[10] M. Koskinen, M. Manninen, S.M. Reimann, Phys. Rev. Lett. 79, 1389 (1997).

[11] T. Ezaki, N. Mori, C. Hamaguchi, Phys. Rev. B 56, 6428 (1997).

[12] H. Tamura, Physica B 249-251, 210 (1998).

[13] O. Steffens, M. Suhrke, U. Rössler, Physica B 256- 258, 147 (1998).

[14] S. Bednarek, B. Szafran, J. Adamowski, Phys. Rev. B 61, 4461 (2000).

[15] S. Bednarek, B. Szafran, J. Adamowski, Microelectron. Eng. 51-52, 99 (2000).

[16] B. Szafran, J. Adamowski, S. Bednarek, Phys. Rev. B 61, 1971 (2000).

[17] L.P. Kouwenhoven, N.C. van der Vaart, A.T. Johnson, W. Kool, C.J.P.M. Harmans, J.G. Williamson, A.A.M. Staring, C.T. Foxon, Z. Phys. B 85, 367 (1991).

[18] J. Weis, R.J. Haug, K. von Klitzing, K. Ploog, Semicond. Sci. Technol. 9, 1890 (1994).

[19] I.-H. Lee, V. Rao, R.M. Martin, J.-P. Leburton, Phys. Rev. B 57, 9035 (1998).

[20] M. Rontani, F. Rossi, F. Manghi, E. Molinari, Phys. Rev. B 59, 10165 (1999).

[21] N.A. Bruce, P.A. Maksym, Phys. Rev. B 61, 4718 (2000).

[22] B. Szafran, J. Adamowski, S. Bednarek, Physica E 5, 185 (2000).

[23] S. Bednarek, B. Szafran, J. Adamowski, Phys. Rev. B 59, 13036 (1999). 
[24] S. Bednarek, B. Szafran, J. Adamowski, to be published.

[25] J. Adamowski, M. Sobkowicz, B. Szafran, S. Bednarek, Phys. Rev. B 62, 4234 (2000).

[26] B. Szafran, S. Bednarek, J. Adamowski, in: Proc. 25th Int. Conf. on Physics of Semiconductors, Osaka (Japan) 2000, Eds. N. Miura, T. Ando, in series Springer Proc. in Physics, Vol. 87, Springer, Berlin 2001, p. 1085.

[27] S. Nagaraja, P. Montagne, V.-Y. Thean, J.-P. Leburton, Y.-H. Kim, R.M. Martin, Phys. Rev. B 56, 15752 (1997).

[28] T.F. Jiang, X.-M. Tong, S.-I. Chu, Phys. Rev. B 63, 045317 (2001).

[29] L.P. Kouwenhoven, D.G. Austing, S. Tarucha, Rep. Progr. Phys. 64, 701 (2001).

[30] R.C. Ashoori, H.L. Störmer, J.S. Weiner, L.N. Pfeiffer, S.J. Pearton, K.W. Baldwin, K.W. West, Phys. Rev. Lett. 71, 613 (1993).

[31] K. Hirose, F. Zhou, N.S. Wingreen, in: Proc. 25th Int. Conf. on Physics of Semiconductors, Osaka (Japan) 2000, Eds. N. Miura, T. Ando, in series Springer Proc. in Physics, Vol. 87, Springer, Berlin 2001, p. 1349.

[32] R.J. Warburton, B.T. Miller, C.S. Dürr, C. Bödefeld, K. Karrai, J.P. Kotthaus, G. Medeiros-Ribeiro, P.M. Petroff, S. Huant, Phys. Rev. B 58, 16221 (1998).

[33] F.M. Peeters, Phys. Rev. B 42, 1486 (1990).

[34] P. Bakshi, D.A. Broido, K. Kempa, Phys. Rev. B 42, 7416 (1990).

[35] M. Fricke, A. Lorke, J.P. Kotthaus, G. Medeiros-Ribeiro, P.M. Petroff, Europhys. Lett. 36, 197 (1996).

[36] P.D. Siverns, S. Malik, G. McPherson, D. Childs, C. Roberts, R. Murray, B.A. Joyce, H. Davock, Phys. Rev. B 58, R10127 (1998).

[37] D.G. Austing, T. Honda, S. Tarucha, Semocond. Sci. Technol. 11, 338 (1996).

[38] J. Schmid, J. Weis, K. Eberl, K. v. Klitzing, Phys. Rev. Lett. 84, 5824 (2000); S. Sasaki, S. De Franceschi, J.M. Elzerman, W.G. van der Wiel, M. Eto, S. Tarucha, L.P. Kouwenhoven, Nature 405, 764 (2000). 